\title{
Evaluation of Automated Guided Vehicle Systems for Container Terminals Using Multi Agent Based Simulation
}

\author{
Lawrence Henesey ${ }^{1}$, Paul Davidsson ${ }^{2}$, Jan A. Persson ${ }^{1}$ \\ Department of Systems and Software Engineering, Blekinge Institute of Technology \\ ${ }^{1}$ Box 214, 37424 Karlshamn, Sweden \\ ${ }^{2}$ Box 520, 37225 Ronneby, Sweden \\ \{lawrence.henesey, paul.davidsson, jan.persson\}@bth.se
}

\begin{abstract}
Due to globalization and the growth of international trade, many container terminals are trying to improve performance in order to keep up with demand. One technology that has been proposed is the use of Automated Guided Vehicles (AGVs) in the handling of containers within terminals. Recently, a new generation of AGVs has been developed which makes use of cassettes that can be detached from the AGV. We have developed an agent-based simulator for evaluating the cassette-based system and comparing it to a more traditional AGV system. In addition, a number of different configurations of container terminal equipment, e.g., number of AGVs and cassettes, have been studied in order to find the most efficient configuration. The simulation results suggest that there are configurations in which the cassette-based system is more cost efficient than a traditional AGV system, as well as confirming that multi agent based simulation is a promising approach to this type of applications.
\end{abstract}

Keywords: MABS application, automated guided vehicles, container terminal

\section{Introduction}

The transport of containers is continuously growing and many container terminals (CTs) are coping with congestion and capacity problems. For instance, the number of Twenty-foot Equivalent Unit containers (TEUs) shipped world-wide has increased from 39 million in 1980 to 356 million in 2004 and growth is projected to continue at an annual rate of 10 per cent till 2020 [1]. Often due to both physical and economic constraints, big container ships are calling on just a few larger ports from which smaller container ships will "feed" containers to other ports in the region. Thus, the number of containers being transhipped is increasing.

There is pressure on the management of ports and CTs to find more efficient ways of handling containers and increase CT capacity. Traditional methods for increasing capacity, such as expanding the CT are often not feasible. Thus, other solutions to increase the efficiency and capacity are considered, including the use of automation, e.g., Automatic Stacking Cranes and Automated Guided Vehicles (AGVs) which may also reduce the operational costs for CTs [3].

The first AGV system was introduced in 1955 for horizontal transport of materials and AGVs were first used for transporting containers in 1993 at the Delta/Sea-Land terminal located in Rotterdam. There has been much research conducted in various areas incorporating AGVs and CTs (cf. Vis [4], for a comprehensive literature review on AGVs). In two European Union sponsored projects; IPSI (Improved Port Ship Inter- 
face) and INTEGRATION (Integration of Sea Land Technologies), a system for handling containers using cassettes and AGVs has been developed but to date has not been used in a CT [5]. The cassettes are steel platforms detachable from the AGV and on which containers can be set. A possible advantage of using cassettes is their ability to act as buffer, since containers can be placed on it without an AGV being present. Figure 1 shows an IPSI AGV and illustrates the different parts of a CT.
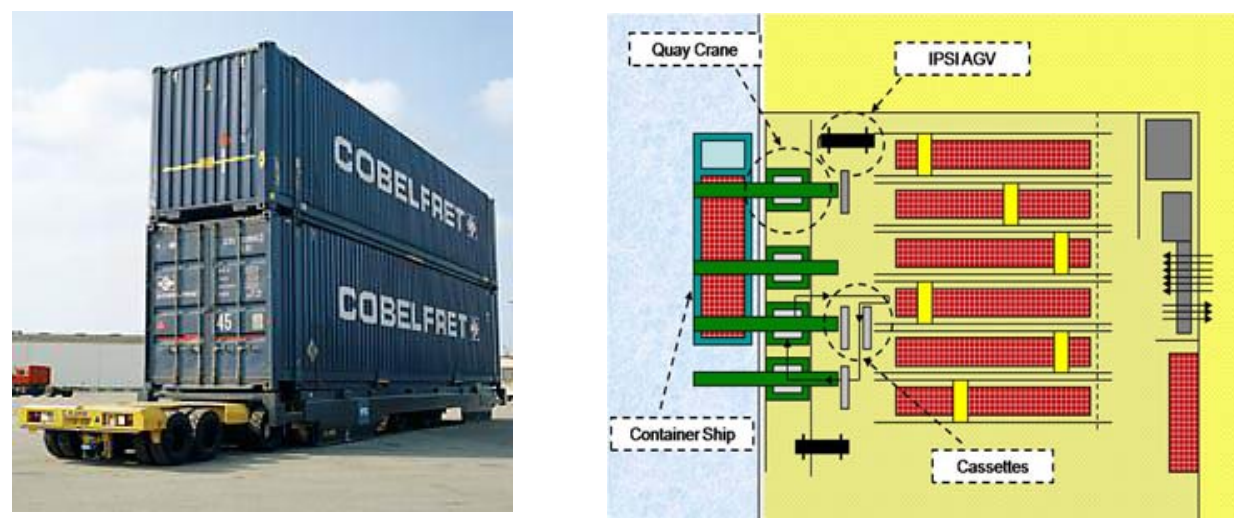

Figure 1. An IPSI AGV transporting a cassette loaded with two containers (left) and a sketch of a container terminal (right).

To evaluate this new development in container handling, we will compare the IPSI AGV system to a "traditional" AGV system, which will be referred to as T-AGV. We perform a comparative analysis of the transport of containers between ship-side operations to the operations in the stacks located in the yard. We also study the effect of the number of cassettes allocated to the IPSI AGV. The developed model can also assist in analyzing aspects, such as, crane assignment to ship and AGV assignment to cranes.

Because of complexity and capital and construction costs, simulation models have been used for understanding behaviour and testing strategies in CTs, e.g., see [6], [7] and [3]. We have developed a multi-agent based simulator (MABS) for comparing the performance of the two AGV systems according to a number of criteria, e.g., service time for a ship, utilization rate for the CT equipment, and operating cost. The choice of using MABS is based on the versatility in simulating complex systems and perceived simplicity in the structure preserving modelling of the different entities in a CT. Parunak et al. [2] compared macro simulation and micro simulation approaches and pointed out their relative strengths and weaknesses. They concluded that “....agentbased modeling is most appropriate for domains characterized by a high degree of localization and distribution and dominated by discrete decision. Equation-based modeling is most naturally applied to systems that can be modeled centrally, and in which the dynamics are dominated by physical laws rather than information processing." As a CT has a high degree of localization and distribution and is dominated by discrete decision, we found agent-based modeling a promising approach worthy to investigate.

The remainder of the paper is organized as follows; in section 2 a description of the problem is provided. In section 3, the methodology and model is presented. Section 4 
provides a description of the simulation experiments. The results are presented and discussed in section 5. Conclusions and future work are provided in section 6 .

\section{Problem Description and Model Assumptions}

A CT is a place where ships will be berthed so that containers can be unloaded and/or loaded by Quay Cranes (QCs). The CT are often viewed as an interface for transport of containers between modes of transport linking the landside with the marine-side [9]. In addition, CTs can serve as temporary storage in the transhipping of containers from one ship to another ship. Ship owners often demand a fast turn-around time since more time for sailing imply more opportunities for extra voyages and more revenue. Further, from a logistics perspective, improving the transport of containers within the CT may help in decreasing the total transport time and cost of transporting cargo in containers. CT managers seek to meet requirements of fast turn-around time and try to optimise the use of their terminal resources, such as, quay cranes for which the capital costs can be $€$ 7 million or more [10], and transporters, such as AGVs. Many CT managers view the interface between the QCs and the yard as the most critical planning problem [11].

In the scenario studied, a ship arrives at a CT with a number of containers to be unloaded and another set of containers are loaded onto the ship before it departs. The unloaded containers are to be transported from the QC area to stacks in the yard and the containers to be loaded are picked-up from stacks and transported to the QC area. We will refer to the QC areas where containers are stored temporarily as the Buffers. The transportation between the buffers and the stacks is carried out by AGVs (either TAGVs or IPSI AGVs with their accompanying cassettes) and we call the time it takes to perform it (including the return without container(s) as well as the load and unloading operations) the AGV cycle time. This definition is similar to the one used in a study comparing a Straddle Carrier system with an Automated Stacking Crane system by Vis [12]. The stacks are located in different areas of the yard and therefore have varying distances to the QC, implying different transport times. We model this by letting the AGV cycle time have random component for each transport. We also consider the time for the unloading and loading of containers from and to a ship by a QC, called the container handling time.

The technical specifications of the two AGV systems considered are presented in Table 1. The IPSI AGV is slightly faster than a T-AGV and has a higher loading capacity as containers cannot be stacked on the T-AGV. The lifting time for an IPSI AGV (the time for it to move under a cassette and lift it off the ground for transporting) is approximately 15 seconds. The T-AGVs do not have a corresponding lifting time since containers are directly loaded on top of them. The initial purchasing cost is provided by industrial partners and serves as an estimate.

Table 1. Specifications of the AGV systems ( $\mathrm{kr}=$ Swedish kronor)

\begin{tabular}{|l|c|c|}
\hline & IPSI AGV & T-AGV \\
\hline Speed (both empty and loaded) & $20 \mathrm{~km} / \mathrm{h}$ & $15 \mathrm{~km} / \mathrm{h}$ \\
\hline Capacity & $82,000 \mathrm{~kg}$ & $55,000 \mathrm{~kg}$ \\
\hline $\begin{array}{l}\text { Maximum container capacity that } \\
\text { can be transported }\end{array}$ & $\begin{array}{c}4 \text { TEU (either } 4 \text { x 20’ } \\
\text { or } 2 \times 40 \text { containers) }\end{array}$ & $\begin{array}{c}1 \text { TEU (one 20' container) or } \\
2 \text { TEU (one 40' container) }\end{array}$ \\
\hline Initial purchasing cost & 4,5 million kr & 2,7 million kr \\
\hline Additional costs (one cassette) & $8,000 \mathrm{kr}$ & Not applicable \\
\hline
\end{tabular}




\section{Methodology and Simulation Model}

In our model there are entities which have a number of attributes and operations associated to them and some of which can communicate with each other. The entities of the real world that we model are: ships, QCs, their buffers, AGVs (IPSI AGV or T-AGV), cassettes and containers. These entities (in some cases including the persons operating them) have to coordinate with each other to complete the main task, which is the unloading/loading a ship. Therefore we model the AGVs, the buffers and the QCs as agents, which. during the simulation perform their task in parallel, replicating the activities in real world CTs. The simulation model was implemented using DESMO-J, an open source library for the JAVA programming language [14]. DESMO-J provides a runtime process based simulation engine that can be used to map port entities to software entities and to simulate the coordination of these process.

The entities use the Contract Net protocol to coordinate tasks. This protocol is used because of its ability to distribute tasks and self-organise a group of agents [15]. The protocol is suitable since our model describes tasks that can be characterised as hierarchical in nature and are well-defined. The Contract Net protocol implies that one agent will take the role of a "manager", which initiates a job to be performed by one or more other agents. The job may require that a number of participating agents respond with a proposal and the manager will accept a proposal and confirm it to a selected agent and reject the other proposals. This protocol seems to closely reflect the operational decisions that are made by the actual workers in the CT, especially when a foreman will communicate via radio with drivers and QC operators. In our case, the buffer agents act as "managers" of the Contract Net protocol and the AGV agents are bidding and performing the jobs.

The system that we have modelled is illustrated in Figure 1 (right-hand side). We have focused on modelling the operations that involves the QCs and the AGVs that transfer containers between the quay and stacks. The main difference between the IPSI $\mathrm{AGV}$ and the T-AGV is the absence of cassettes. The containers need to be placed directly on the AGV in the T-AGV system. A QC will wait for an available T-AGV to place a container on it rather than placing the container on the ground and then having to later pick it up and place it on a T-AGV, in order to reduce extra moves by the QC. We have followed a general simulation process as described by Law and Kelton [8] and therefore we are testing a prototype with real data.

The entities that are modelled are:

- Ship: contains the containers.

- Quay cranes: used to unload and load the containers.

- AGVs (IPSI AGVs or T-AGVs): used to transport container, from/to QC from/to a container stack.

- Buffer: Pick-up and drop-off area behind the QC that temporarily stores containers on cassettes or AGVs.

- Cassettes: A set of cassettes bound to a QC.

- Containers

As mentioned earlier, the QCs, Buffers and the AGVs are modelled as agents, whereas the ships and cassettes are modelled as objects. 


\subsection{Quay Crane (QC) Agent}

The QC is responsible for the unloading and loading the containers from and to the ship. The number of QCs serving a ship is specified by the user. Each QC has the following attributes:

- $\quad$ a set of AGVs (IPSI AGVs or T-AGVs) assigned to it

- a set of cassettes (for the IPSI AGVs)

- a buffer area where its cassettes or T-AGVs can be placed for loading/unloading containers

- container handling time for (un)loading a container. In a real CT the container handling time will vary for each container, which we simulate by a computergenerated random number using a linear congruence method chosen in a range specified by the user.

Moreover, the following are recorded for each QC:

- the number of containers unloaded from the ship and loaded to the ship

- the time it has been working (not including the idle time).

A QC performs the following functions

- When unloading, the QC will unload a container from the ship if there is a cassette (or T-AGV) with free space in the buffer area. If not, it waits until there is one available.

- When there are containers to be loaded available in the buffer area, the QC will load one container at a time to the ship.

- The QC finishes working when there are no containers to be unloaded or loaded for the ship.

\subsection{Buffer Agent}

A buffer is assigned to a specific QC and the buffer is responsible for allocating free AGVs to either pick-up a container and move it or move empty (to pick-up container(s) at another location). The buffer is also responsible for the QC to stop unloading if there is no cassette available or the cassette is full and to stop loading if there is no containers available on cassettes or on AGVs. The Buffer agent will communicate with the AGVs and assign an AGV that is free to pick up a cassette (for the IPSI-AGV). Once a cassette is available the buffer agent will ask the QC to start working. When using TAGVs, the buffer agent tries to find a free T-AGV and assign a container to that $\mathrm{T}$ $\mathrm{AGV}$. If no T-AGV is free then it waits until a T-AGV is free at the buffer. Pseudocode describing the unloading and dispatching strategy of the buffer agent for the cassette-based system is given below:

WHILE still containers to unload DO

IF cassette available that has room for more containers THEN

Ask QC to unload a container and place it on cassette

Ask all AGVs for their status

Wait for status reports

IF AGV idle THEN

Ask that AGV to fetch the loaded cassette

ELSEIF cassette is full THEN

Ask QC to stop unloading 
REPEAT

Ask all AGVs for their status

Wait for status reports

UNTIL at least one AGV is idle

Ask the idle AGV to fetch the loaded cassette

ENDWHILE

\subsection{AGV Agent (IPSI AGV and T-AGV)}

Each QC has a number of AGVs assigned to it. This value is specified by the user before the start of the experiment. An AGV is responsible to transport containers between a QC Buffer and container stacks. Each AGV has the following attributes:

- a state ("free" or "busy")

- a cycle time for transporting a cassette/container from the buffer to the stack and return back to the buffer. Or vice versa during the loading phase. In a real CT the AGV will have varying transport times, which we simulate by a computer generated random number using a uniform method chosen in a range specified by the user.

Moreover, the following are recorded for each AGV:

- the number of containers transported to/from the stack

- the time it has been working (not including the idle time).

\section{Experiment Description}

The input parameters are stored in a text file from which the simulator reads the parameters. The output of the simulation is a set of files which contains information of all events taken place during the simulation. A trace file contains the overall performance of each QC, AGV and cassette involved in the simulation. The performance criteria that are used for evaluating and comparing the CT transport systems are:

- Service Time: is the time it takes to complete the unload/load operations for a ship, also known in the maritime industry as "turn-around time".

- Utilization Rate: Active time / Service Time (Active time + Idle time). Active time is the time a piece of CT equipment is busy, e.g. moving a container from the QC to a stack, and Idle time is the time that it is not working. The utilization rate for the following equipment is recorded: $Q C, A G V$ and Cassette.

- Throughput: Avg. number of containers handled per hour during Service time for: QC, AGV and Cassette

- Total Cost: Equipment cost for serving a ship is calculated in the following ways (OPEX = operating cost per hour for a unit of CT equipment):

- QC: number of QCs x OPEX for QC x Service Time.

- AGV: number of AGVs x OPEX for AGV x Service Time.

- Cassette: number of cassettes x OPEX for Cassette x Service Time.

- Total Cost: QC costs +AGV costs + Cassette costs

\subsection{Scenario Settings}

The scenario settings were based upon data provided by industrial partners. The results from the simulations are average values. Thus, a sufficient number of runs are needed 
in order to get a valid estimation. The cycle times used in the simulation have been determined from prior analysis in which the stack distances and maximum speeds of the AGVs were tested. We used an approximation method to calculate the minimum number of simulation runs required in order to obtain results from a simulator with small enough statistical errors. The approximation method [16] has been applied by Vis et al. [17] in vehicle allocation at a container terminal. In this method, data from a limited number of replications is used to approximate the required minimum number of replications in the actual experiment (denoted $\mathrm{i}$ ) such that the relative error is smaller than $\gamma(0<\gamma<1)$ with a probability of $1-\alpha$. The i value can be calculated from:

$$
i \geq S^{2}(i)\left[z_{1-\alpha / 2} / \gamma^{\prime} \bar{X}(i)\right]^{2}
$$

where $S^{2}(i)$ is the variance of the trial sample, $Z_{1-\alpha / 2}$ is the $1-\alpha / 2$ percentile of the normal distribution, $\bar{X}(i)$ is the trial sample mean value, and $\gamma^{\prime}=\gamma /(1+\gamma)$. Based on our trial sample we found that to obtain the error smaller than $2 \%(\gamma<0.05)$ with a probability of $95 \%$ that the number of generated replications would be sufficient at 100 for all experiments conducted in this paper. In the simulation experiments, we use the settings listed in Table 2 for serving a single ship.

Table 2. Settings experimented in the simulator for a single ship

\begin{tabular}{|l|c|c|}
\hline \multicolumn{1}{|c|}{ Input Settings for Scenario } & \multicolumn{2}{c|}{ AGV Type } \\
\hline No. Containers & IPSI AGV & T-AGV \\
\hline No. QCs & 493 & 493 \\
\hline No. Cassettes assigned to an QC for each IPSI AGV & 3 & 3 \\
\hline No. AGVs per QC & $1-4$ & n.a. \\
\hline Container handling time for QC & $1-5$ & $1-5$ \\
\hline Travel cycle time for AGV & $1-2 \mathrm{~min}$ & $1-2 \mathrm{~min}$ \\
\hline
\end{tabular}

An "average ship" is used in which 493 containers are to be either unloaded or loaded, and there are 3 QCs to serve the ship. Each QC is assigned 1 to 5 AGVs and has a container handling time randomly generated for each container ranging between 1 to 2 minutes. The numbers of cassettes are from one to four per IPSI AGV. Each IPSI AGV has a travel cycle time that is randomly generated for each cassette transported ranging between 3 to 6 minutes. The T-AGVs posses a random travel cycle time ranging between 3 to 5 minutes. Cycle time for the IPSI AGV includes the lifting of a cassette, transport it from a QC to a stack, detach the cassette and then return to the QC with an empty cassette; or the cycle time for the opposite direction, i.e., transporting from the stack to the QC. Cycle time for T-AGVs is similar to IPSI AGVs but does not have a lifting time or transport a cassette.

\section{Simulation Results}

Simulation experiments were conducted to evaluate various combinations of allocated terminal resources three QCs. Ship service time results are presented in Figure 2, for different number of AGVs and cassettes used (the exact values are given in Table 3). They suggest that ship service time is generally faster for IPSI AGVs than for T-AGVs. 
When three or more IPSI AGVs with two or more cassettes each, the service time is close to its smallest value and instead the capacity of the QCs becomes the bottleneck. Ship service time results for the T-AGV system are similar to the IPSI AGVs when assigned with one cassette. The ship service time appears to be faster after two IPSI AGVs are assigned with two or more cassettes, average ship service time is 5,13 hours. The fastest ship service time is 4,10 hours when using four cassettes and either four or five IPSI AGVs. The use of an additional IPSI AGV when using four cassettes appears not to influence the ship service time.

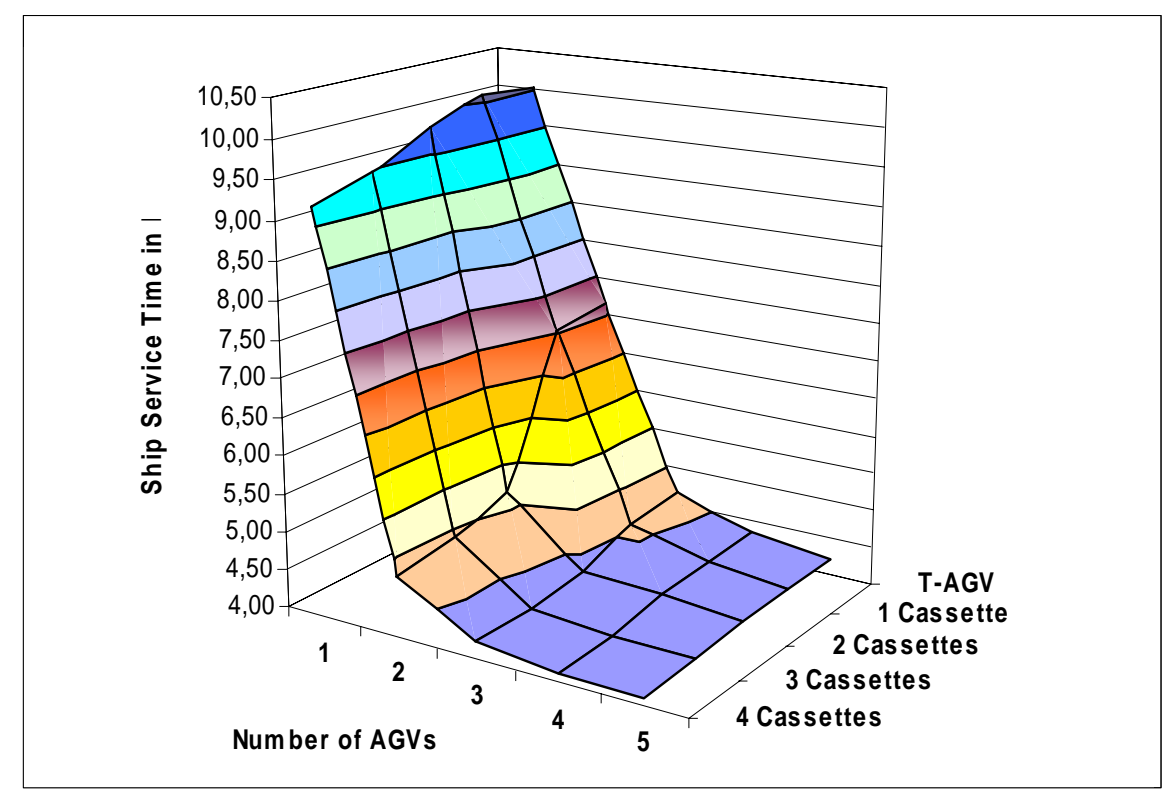

Figure 2. Simulation results for ship service time.

Table 3. Average ship service times (and standard deviation).

\begin{tabular}{|c|c|c|c|c|c|}
\hline No. AGV & T-AGV & 1 Cassette & 2 Cassettes & 3 Cassettes & 4 Cassettes \\
\hline 1 & $10,03(0,14)$ & $10,08(0,12)$ & $9,85(0,13)$ & $9,45(0,14)$ & $9,23(0,13)$ \\
\hline 2 & $7,15(0,05)$ & $7,03(0,06)$ & $5,13(0,06)$ & $4,90(0,07)$ & $4,78(0,06)$ \\
\hline 3 & $4,65(0,06)$ & $4,58(0,07)$ & $4,32(0,05)$ & $4,23(0,06)$ & $4,23(0,06)$ \\
\hline 4 & $4,33(0,06)$ & $4,30(0,06)$ & $4,27(0,07)$ & $4,15(0,06)$ & $4,10(0,06)$ \\
\hline 5 & $4,20(0,06)$ & $4,20(0,06)$ & $4,18(0,06)$ & $4,13(0,07)$ & $4,10(0,06)$ \\
\hline
\end{tabular}

From the simulation experiments, we can compare the QC utilization rates in Table 4. Generally, we see that the more transport equipment is available, the higher is the QCs' rate of utilization. The rate of QC utilization becomes close to one when using three or more IPSI AGVs with at least two cassettes per AGV. 
Table 4. Comparison of QC Utilization Rates

\begin{tabular}{|c|c|c|c|c|c|}
\hline No. AGVs & T-AGV & 1 Cassette & 2 Cassettes & 3 Cassettes & 4 Cassettes \\
\hline 1 & 0,41 & 0,41 & 0,42 & 0,43 & 0,44 \\
\hline 2 & 0,57 & 0,58 & 0,80 & 0,84 & 0,86 \\
\hline 3 & 0,88 & 0,89 & 0,95 & 0,97 & 0,97 \\
\hline 4 & 0,95 & 0,95 & 0,96 & 0,99 & 1,00 \\
\hline 5 & 0,98 & 0,98 & 0,98 & 0,99 & 1,00 \\
\hline
\end{tabular}

AGV utilization rates are presented in Table 5. We see that the utilization rate is close to 1 when only one AGV is used, that is, the QC is able to keep the AGV busy. When more AGVs are added, the utilization rate decrease and the AGVs spend more time being idle. In comparing T-AGVs with the IPSI AGVs, there is a recorded higher level of utilization when IPSI AGVs each have two or more cassettes. Utilization rates for the IPSI AGVs decrease in smaller increments as the number of cassettes increase.

Table 5. Comparison of AGV Utilization Rate (IPSI AGV and T-AGV)

\begin{tabular}{|c|c|c|c|c|c|}
\hline No. AGVs & T-AGV & 1 Cassette & 2 Cassettes & 3 Cassettes & 4 Cassettes \\
\hline 1 & 0,957 & 0,962 & 0,995 & 0,997 & 0,991 \\
\hline 2 & 0,687 & 0,673 & 0,939 & 0,971 & 0,983 \\
\hline 3 & 0,659 & 0,680 & 0,730 & 0,755 & 0,744 \\
\hline 4 & 0,543 & 0,559 & 0,570 & 0,575 & 0,578 \\
\hline 5 & 0,477 & 0,484 & 0,476 & 0,469 & 0,468 \\
\hline
\end{tabular}

An increase in the number of cassettes and AGVs adds extra capacity for transporting containers. The extra capacity provided by cassettes may be viewed as a 'floating buffer', which allows the IPSI AGVs to decouple the load of containers on a cassette and fetch another cassette. This activity assists in lessening the idle time of the QCs so that they can be more productive. Thus, from the above results one can conclude that it is useful to introduce a certain amount of IPSI AGV and cassettes in the simulation to make the crane busy throughout the simulation. As crane operating cost is higher than the AGV operating cost, these results can be helpful for CT management in deciding, e.g., how many cranes, IPSI AGVs and cassettes to be allocated to a ship. We shall now compare operating costs for the different configurations.

In Table 6 and Figure 3 the total operating costs for employing the CT equipment is presented. In determining the total operating costs the hourly operating cost is multiplied by the number of CT equipment type employed, which is then multiplied by ship service time. The assumed hourly operating costs (including depreciation, maintenance, labour and fuel) used in the calculations are (in Swedish kronor per hour):

- QC: $905 \mathrm{kr} / \mathrm{hour}$

- T-AGV: 43 kr/hour

- IPSI-AGV: 60 kr/hour

- Cassette: 0,50 kr/hour 


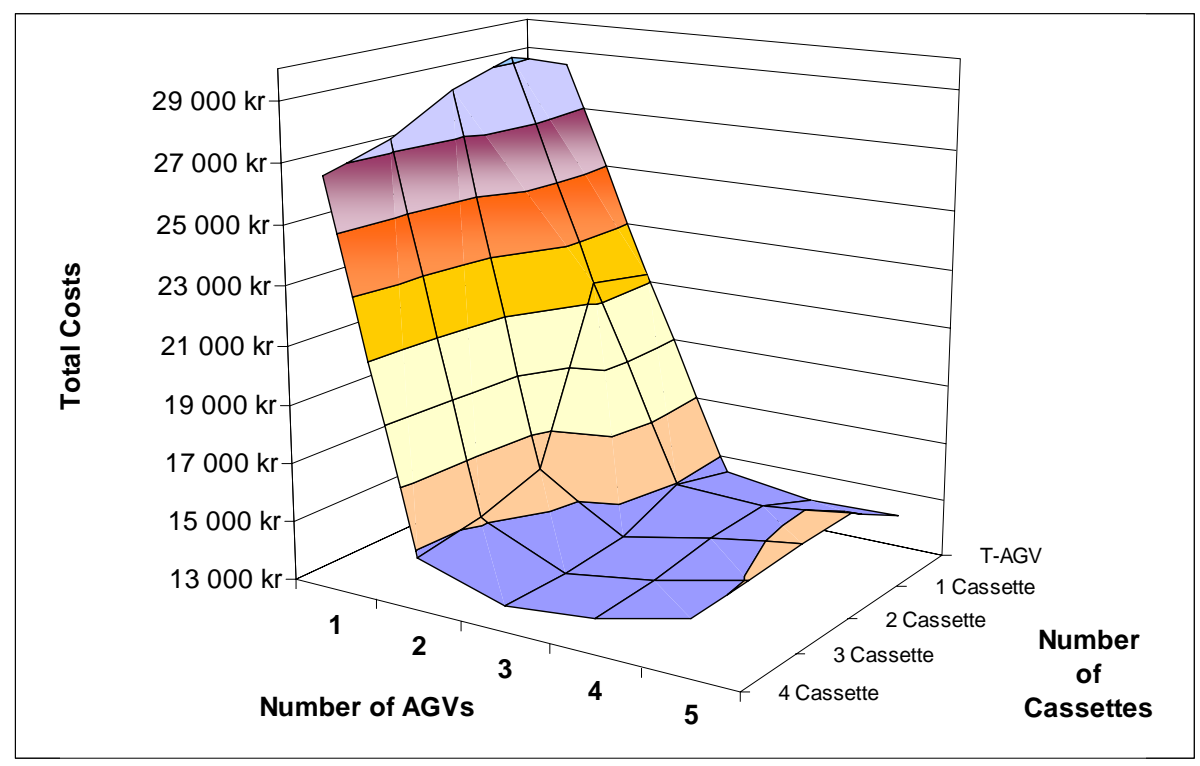

Figure 3. Total operating costs for serving a ship.

Table 6. Total operating costs for serving a ship.

\begin{tabular}{|c|c|c|c|c|c|}
\hline No. of AGVs & T-AGV & 1 Cassette & 2 Cassettes & 3 Cassettes & 4 Cassettes \\
\hline 1 & $28535 \mathrm{kr}$ & $29206 \mathrm{kr}$ & $28545 \mathrm{kr}$ & $27400 \mathrm{kr}$ & $26786 \mathrm{kr}$ \\
\hline 2 & $21257 \mathrm{kr}$ & $21649 \mathrm{kr}$ & $15816 \mathrm{kr}$ & $15112 \mathrm{kr}$ & $14766 \mathrm{kr}$ \\
\hline 3 & $14424 \mathrm{kr}$ & $14939 \mathrm{kr}$ & $14090 \mathrm{kr}$ & $13837 \mathrm{kr}$ & $13856 \mathrm{kr}$ \\
\hline 4 & $14001 \mathrm{kr}$ & $14796 \mathrm{kr}$ & $14707 \mathrm{kr}$ & $14330 \mathrm{kr}$ & $14182 \mathrm{kr}$ \\
\hline 5 & $14112 \mathrm{kr}$ & $15215 \mathrm{kr}$ & $15186 \mathrm{kr}$ & $15035 \mathrm{kr}$ & $14945 \mathrm{kr}$ \\
\hline
\end{tabular}

In comparing the total operating costs, the addition of more AGVs and cassettes leads to lower costs up until three IPSI AGVs and three cassettes are employed. The total costs when adding further equipment increase, but the time gained do not compensate for the extra cost. Thus, from a cost perspective the best choice in the scenario studied would be to use three IPSI AGVs with three cassettes each. From a ship service time perspective, on the other hand, an additional AGVs and an additional cassette for each AGV would give even better results (but inly slightly). Thus, a CT manager would have to make the final decision of how to balance the quality of service provided with the total costs.

\section{Discussion, Conclusion and Future Work}

The simulation experiments have provided much insight into the properties of various terminal equipment types that can be used in container terminal operations. The cassette-based system posses some advantages in that it can act as a 'floating' buffer, meaning that it can allow the QCs to keep unloading/loading and not having to wait for an AGV to be available. Waiting time is lower for the QCs and thus they are obtaining better utilization rates. The initial results from the prototype AGV simulator provide some interesting observations useful for determining the number of machinery units to 
allocate for serving a ship. The simulation experiments that we have conducted are also creating further questions that require more investigation. Naturally there is a trade-off to be expected between service time and the costs for purchasing and operating equipment.

Compared to traditional simulation approaches, the MABS approach can provide finer granularity in modelling the entities and having them communicate and coordinate amongst other entities. In particular, we found this useful in the studied application where the characteristics of different types equipment need to be captured, and where equipment is distributed in physical space and the need to coordinate their activities is essential. More generally, we argue that MABS seems a promising technology for evaluation and comparison of different automation approaches. Elder [13] mentions some general advantages of simulation methods over applying queuing theory. In addition, we found it difficult to use queuing theory to model activities such as the AGV dispatching strategies (in this study carried out by the Buffer agent) that are used when applying cassette-based systems.

For future work we would study other models for container handling time by the QC and the cycle time for AGVs (e.g., including stoppages caused by malfunctioning equipment, etc. which affects the productivity at a real CT). Another topic worth studying is different dispatching strategies for allocating containers to AGVs and cassettes. We plan to extend the model in several directions, e.g., including the unloading/loading taking place at the stacks, more detailed modelling of the AGV movements, etc.

\section{Acknowledgements}

This work has been partially funded by Karlshamn Municipality. The following port industry representatives have provided useful information necessary for the development of IPSI AGV Simulator; TTS AB in Göteborg, Sweden, Lennart Svensson, Bjørn O. Hansen and Michel Lyrstrand. Much appreciation is given to Khurum Aslam for related work with the DESMO-J simulator and to Dr. Piotr Tomaszewski.

\section{References}

1. Davidson, N. A global capacity assessment and needs analysis. In: 39th Terminal Operating Conference. Antwerp, Belgium: Informa Plc., 2005.

2. Parunak, H. V. D., Savit, R., and Riolo, R. L.: Agent-Based Modeling vs. EquationBased Modeling: A Case Study and Users' Guide. In: Multi-Agent Systems and Agent-Based Simulation, LNAI, Vol. 1534, Springer, pp. 10-26, 1998.

3. Ioannou, P.A., Kosmatopoulos, E.B., Jula, H., Collinge, A., Liu, C.-I., Asef-Vaziri, A., Cargo Handling Technologies. Technical Report. Department of Electrical Engineering, University of Southern California: Los Angeles, pp. 1-147, 2002.

4. Vis, I.F.A., Survey of research in the design and control of automated guided vehicle systems. European Journal of Operational Research, 170(3), pp. 677-709, 2006.

5. TTS AB Port Equipment; http://www.tts-marine.com.

6. Bruzzone, A.G., Giribone, P., and Revetria, R. Operative requirements and advances for the new generation simulators in mulitmodal container terminals. In: Proceedings of the 1999 Winter Simulation Conference. Society for Computer Simulation International, 1999. 
7. Hayuth, Y., M.A. Pollatschek, and Y. Roll, Building a Port Simulator. Simulation, 63(3) pp. 179-189, 1994.

8. Law, A.M. and W.D. Kelton, Simulation Modeling and Analysis. 3rd ed., Boston: McGraw-Hill International, 2000.

9. Rida, M., Boulmakoul, A., Laurini, R., Calibration and validation of container terminal simulation. In: Proceedings of the 3rd IEEE International Symposium on Signal Processing and Information Technology, pp. 774-777, 2003.

10.De Monie, G., Environmental Scanning in Ports, In: ITMMA Private Public Partnerships in Ports, Antwerp, Belgium, 2005.

11.Henesey, L., Davidsson, P., and Persson, J.A., Agent Based Simulation Architecture for Evaluating Operational Policies in Transshipping Containers. Multiagent System Technologies. LNAI, Vol. 4196, Springer, pp. 73-85, 2006.

12.Vis, I.F.A., A comparative analysis of storage and retrieval equipment at a container terminal. International Journal of Production Economics, 103(2) pp. 680-693, 2006.

13.Elder, M., Simulation beats Queuing, SIMUL8, 2005.

14.DESMO-J, http://www.desmoj.de/

15.Clearwater, S.H., Market-Based Control: A Paradigm for Distributed Resource Allocation, Singapore: World Scientific, 1996.

16.M. Law, A. and W.D. Kelton, Simulation Modeling and Analysis., Boston: McGraw-Hill International, 2000.

17.Vis, I.F.A., R. de Koster, and M.W.P. Savelsbergh, Minimum vehicle fleet size under time-window constraints at a container terminal. Transportation Science, 39(2), pp. 249-260, 2005. 